\title{
Study on the Subclavian Artery and its Branches in Japanese Adults
}

\author{
By \\ Toyoharu TAKAFUJI and Yasushi SATO \\ First Department of Anatomy, Kyorin University School of Medicine, 20-2, Shinkawa 6-chome, Mitaka-shi, Tokyo 181, \\ Japan. \\ - Received for Publication, May 24, 1991 -

\begin{abstract}
Key Words: Subclavian artery, Thyrocervical trunk, Transverse cervical artery, Suprascapular artery, Dorsal intersegmental arteries

Summary: We examined the entire array of branches and the state of ramification of each branch of 144 subclavian arteries $(\mathrm{Su})$ in 72 Japanese adults, and obtained the following findings. (1) The incidence of Type $I-A(1)$ was $13.2 \%$, the highest among the entire $S u$ ramification. This was followed by Type $I-B(1)$ at $9.0 \%$, then Type $I-A(2)$ at $6.9 \%$, indicating considerable variation in the morphology of $S u$ ramification. (2) Ontogenetic factors were implicated because of the existence of cases in which $S u$ traversed the scalenus anterior muscle anteriorly (Type III: $0.7 \%$ ), or was transfixed $(0.7 \%)$. (3) Type 1-c was the form of thyrocervical trunk $(\mathrm{Tt}$ ) observed with the highest incidence of $31.3 \%$. In addition, we propose that Types $a-j$ (that were formed from a common trunk consisting only of the ascending cervical artery and inferior thyroid artery, and those in which another branch was included in this common trunk: $88.9 \%$ ) be called $T$ tc. (4) The most common type of transverse cervical artery $(T c)$, which is formed from a common trunk consisting of superficial and deep branches, was found to be Type 1-a that branches from the first part of Ttc. It was observed with the highest incidence of $61.8 \%$. Some of the superficial branches to the upper portion of the trapezius muscle that were independent and branched from Ttc (Types $e, f: 2.8 \%$ ), we called upper superficial branches. (5) The most frequently observed type of suprascapular artery $(S s)$ was Type $b(38.2 \%)$, followed by Type $a(25.7 \%)$, which branches independently from $\mathrm{Su}$. We concluded that the sites at which $\mathrm{Tc}$ and $\mathrm{Ss}$ originate are the positions at which they traverse the brachial plexus (superior, transfixed to the plexus). (6) In cases in which the supreme intercostal artery (Is) and the deep cervical artery $(C p)$ were separate, (Types $d-i$ excluding Type $g: 31.9 \%$ ), Is branched from a more proximal position than $C p$.
\end{abstract}

Formation of the human subclavian artery to an almost adult condition occurs in about 7 weeks of embryonic development. ${ }^{5,6,10,26)}$ There have been numerous reports of substantial individual and racial differences in the position of arterial branches and the nature of their ramifications as a result of various factors that have an influence on morphosis. . $\left.^{12,13}, 17,21,27,29,33,35,44\right)$ However, there are almost no reports of studies that have attempted to characterize and classify the ramification and positions of the entire array of branches arising from the subclavian artery. The only relevant reports in Japan were those by Adachi $\left({ }^{\prime} 28\right)^{1)}$ and Ouchi $\left({ }^{\prime} 63\right)^{30)}$, who described the subclavian artery ramification in Japanese adults, and that by Mori $\left({ }^{\prime} 41\right)^{28)}$, who described Japanese fetuses. However, these reports did not explain the entire subclavian artery ramification, nor determined the most common type of ramification or its incidence among the Japanese population.

With recent increase in the use of CT, MRI and angiography, it has been pointed out that an accurate understanding of the morphology of the normal sub- clavian artery ${ }^{32)}$ and detailed information about the ramification and course of arteries that branch from the subclavian artery ${ }^{12.22)}$ are essential prerequisites for accurate diagnosis by these techniques of pathogenic changes in the upper limbs. Furthermore, sternocleidomastoid muscle and trapezius muscle are frequently used as a musculocutaneous flap in reconstructive surgery, but despite the fact that the nutrient artery which supplies these muscles is a branch of the subclavian artery, reports to the contrary have appeared. ${ }^{31,40)}$

For these reasons, we conducted a study to characterize and classify the entire ramification of branches arising from the subclavian artery, as well as the ramification and position of the sites of origin of the individual branches in Japanese subjects. We also determined the incidence of the different types found in the study. 


\section{Method and Materials}

\section{Materials}

144 subclavian arteries of 72 Japanese adults (43 males and 29 females) were examined. The mean age of the subjects was 71.2 years (45-92 years).

\section{Method}

The entire subclavian artery and its branches were excised with care, examined in detail, and a sketch made of the sites of origin, course, and termination of each branch. We also classified the subclavian artery into the 1st part (medial to the scalenus anterior muscle), 2nd part (posterior to the muscle) and 3rd part (lateral to the muscle), based on its position relative to the scalenus anterior muscle. We named each of the arteries by following their course and distribution in the muscles.

\section{Results}

Fig. 1 shows the right subclavian artery $(S u)$ and its branch ramification of a 71-year-old female (No. $315 \mathrm{R})$. The vertebral artery (Ve) had an outer diameter of $3.1 \mathrm{~mm}$, originated from the $1 \mathrm{st}$ part $1.5 \mathrm{~cm}$ lateral to the branching portion of the subclavian artery, and extended to the transverse foramen of the 6th cervical vertebra posteromedially. At a site $5 \mathrm{~mm}$ lateral to $V e$, the thyrocervical trunk (Ttc) that originated superiorly from the 1 st part immediately proximal to the medial border of the scalenus anterior muscle ( $s a)$, branched to form the suprascapular artery $(S s)$ at approximately $1 \mathrm{~cm}$ posterior to the branch. Ss traversed the anterior of $s a$ laterally, but after passing this muscle, its direction became slightly more posterior, passing superior to the brachial plexus $(P l b)$, with one branch feeding the clavicular portion of the trapezius muscle. After passing above the superior scapular transverse ligament of the scapula, Ss traversed behind the supraspinatus muscle, passed around the neck of the scapula, then branched to supply a fine vessel to the superior border of the posterior portion of the deltoid muscle, and another to the supraspinatus muscle ventrally. Intramuscular distribution in the trapezius muscle and deltoid muscle was equivalent to Type II- $a$ $\left(\right.$ Takafuji $\left.{ }^{43)}\right)$ and Type 1-s $\left(\right.$ Azuma $\left.^{4)}\right)$ as previously reported.

The transverse cervical artery $(T c)$ branched directly from $T t c$ at a position $5 \mathrm{~mm}$ distal from $S s$, then traversed the anterior $s a$ laterally, passing over $P l b$ to reach the lateral border of the levator scapulae muscle, where it split into a superficial branch $(R s)$ and deep branch $(R p)$. Rs extended to the middle

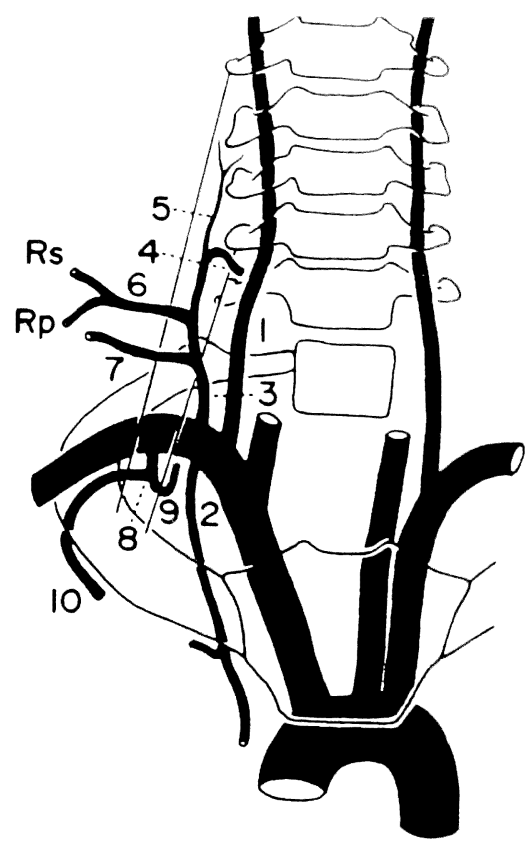

Fig. 1. Schematic drawing of the subclavian artery and its branches on the right side of a 71 year-old Japanese female (No. 315). This is the typical type (I-A(1)) in this series.

Abbreviations for Figures

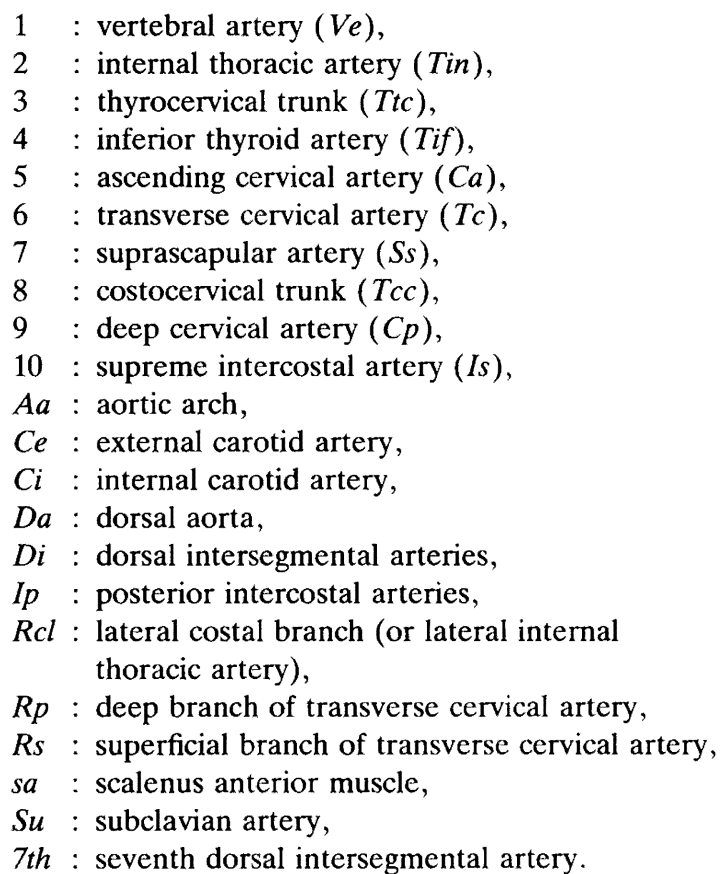

portion of the trapezius muscle along the dorsal side of the levator scapulae muscle (superficial layer), then immediately before penetrating the muscle, split into an upper branch that supplied the upper muscle bundle (clavicular portion) and a middle branch that supplied the middle portion of the muscle (acromial portion/portion of the spine of the scapula). After branching, $R p$ supplied the rhomboid and serratus anterior muscles dorsally after passing under the ventral sides (deep layers) of the levator scapulae muscle and the rhomboid muscle along the 
medial border of the scapula. In the course of this inferior passage, $R p$ sends a branch $(B l p)$ to the lower portion of the trapezius muscle. $B l p$ penetrates the trapezius muscle after becoming transfixed between the rhomboideus major and minor muscles. Immediately after $T c$ bifurcates, $T t c$ splits into the ascending cervical artery $(\mathrm{Ca})$ and the inferior thyroid artery (Tif). $\mathrm{Ca}$ supplies this muscle with 3 fine branches during its ascending course on the anterior surface of $s a$, then penetrates the intervertebral foramen along the 4th or 5th cervical nerve. Tif follows an inner descending course to reach the center of the external border of the thyroid gland, supplies one fine branch to the esophagus, then descends posteromedially to supply the inferior half of the thyroid gland.

The internal thoracic artery (Tin) followed an inferomedial course after branching inferiorly from the 1st part of $S u$ in the reflective direction from Ttc. It then followed a descending course approximately $1.5 \mathrm{~cm}$ external to the outer edge of the sternum, and supplied anterior intercostal branches and perforating branched to each intercostal space. After this, Tin branched to form the musculophrenic artery and the superior epigastric artery at the inferior edge of the sternum. The costocervical trunk $(T c c)$ arose from the dorsal surface of the 2nd part of $S u$, then immediately branched into the deep cervical artery $(C p)$ and the supreme intercostal artery $(I s) . C p$, after running dorsally sent one fine branch to the intervertebral foramen along with the 8th cervical nerve. The other branch supplied the splenius muscle after following an ascending course. Is sent a branch to the 1 st and 2 nd intercostal space after following a descending course after branching.

The following describes the sites of origin and the morphology of ramification of the subclavian artery $\mathrm{Su}$ ) and each artery that branches from it in all of the subjects examined.

\section{1) Relation between $S u$ and $s a$}

Fig. 2 shows the positional relation of the subclavian artery $(S u)$ to the scalenus anterior muscle (sa). In 142 among 144 arteries, $S u$ traversed sa posteriorly (Type I: $98.6 \%$ ), but in one case (a 76year-old male; left side) it was transfixed to $s a$ (Type II: $0.7 \%$ ), and in another case (a 79-year-old male; left side) it traversed $s a$ anteriorly (Type III: $0.7 \%$ ).

\section{2) $\mathrm{Ve}$}

Fig. 3 shows the site of origin and the morphology of ramification of the vertebral artery $(V e)$. Type $A$, in which the left vertebral artery branches directly from the aortic arch $(A)$, was observed in 2 males and 3 females, a total of 5 subjects. The incidence of Type $A$ was $6.9 \%$ by subject, and $3.5 \%$ by body side
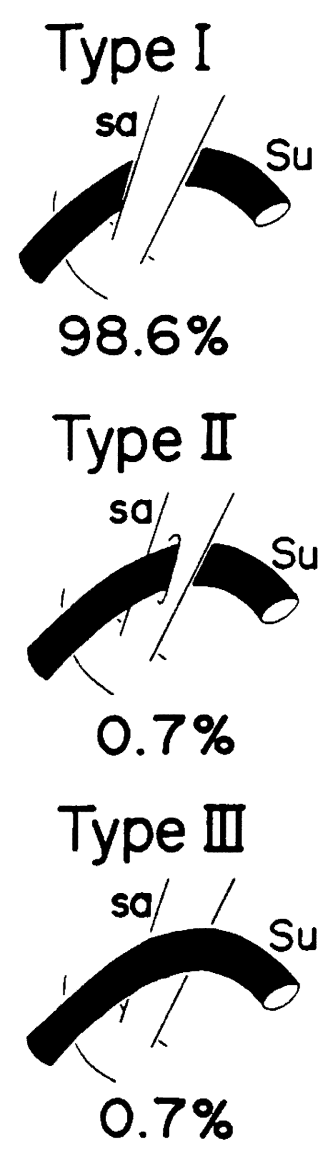

Fig. 2. Three positional relations of the subclavian artery $(S u)$ to the scalenus anterior muscle.

of appearance.

Additional classifications were combinations of the following two types (classification). Types $A, 1$, 2, 3, and $A x$ indicate the site at which the artery arose, $(A)$ representing branching from the aortic arch, 1, 2, and 3 from the 1st, 2nd, and 3 rd parts of the subclavian artery, and $A x$ from the axillary artery. Types represented by the letters $a-q$ represent the nature of ramification (indicating whether or not the artery forms a common trunk with another branch) at the origin of each artery.

The most common type of $V e$ was Type $a(93.1 \%)$, in which $V e$ branched independently and medially from the thyrocervical trunk (Ttc). A common form of Type $a$ was Type 1- $a$ in which branching was from the 1st part of $S u$ in 129 cases or $89.6 \%$ (males: $89.5 \%$, females: $89.7 \%$, right: $87.5 \%$, left: $91.7 \%$ ). Type 1-d, in which $V e$ formed a common trunk with Tif, was also observed in 2 cases $(1.4 \%)$.

\section{3) Tin}

The internal thoracic artery $(\mathrm{Tin})$ followed $V_{c}$ in showing branching from a fairly consistent position (Fig. 4). Type $a$ showing independent branching of Tin was seen in 126 cases $(87.5 \%)$. Type $1-a$, in which formation of Tin from the first part of $S u$. was observed in $79.9 \%$ (males: $80.2 \%$, females: $79.3 \%$, 


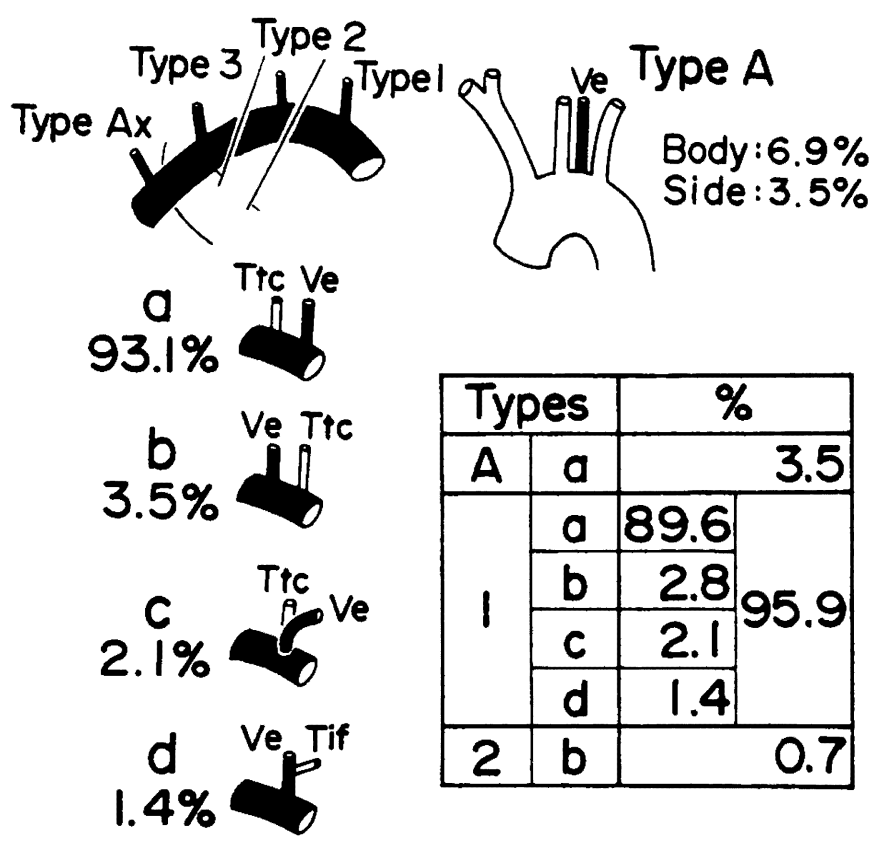

Fig. 3. Types and incidences of origin of the vertebral artery ( Ve) classified by the site and the forming of common trunk with the other artery. For descriptive purposes, the subclavian artery was divided by the scalenus anterior muscle into 3 portions: medial to it (1st part), behind it (2nd part) and lateral to it (3rd part). In all figures of this study, additional classifications were combinations of the following two types. Types $A, 1,2,3$, and $A x$ indicate sites of origin for each artery, which arose from the aortic arch, the 1st part, the 2nd part and the 3rd part of the subclavian, and axillary artery, respectively; Types $a \sim q$ indicate their trunk formation with the other arteries.

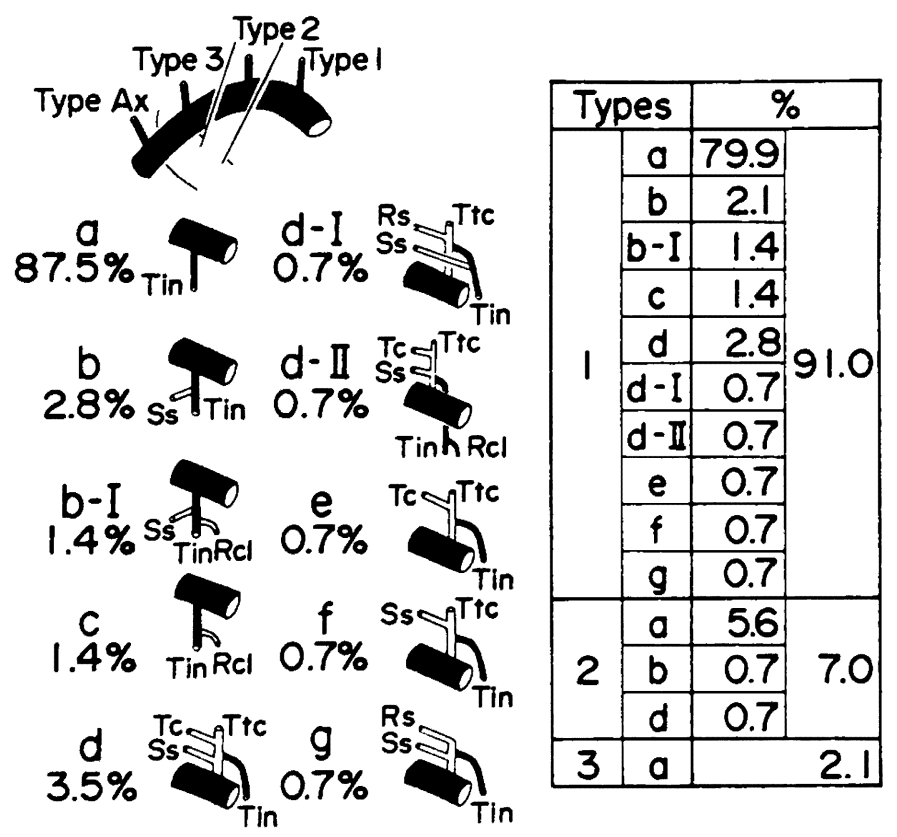

Fig. 4. Types of the origin of the internal thoracic artery (Tin) classified by their site and how they form common trunk with the other arteries. In Types $b$-I, $c$ and $d$-II, the lateral costal branch $(R c l)$, occurred as a variational artery, arose from Tin which originated either alone or as a common trunk from the subclavian. These types were encountered in $3.5 \%$ of cases, and especially Type $d$-II $R c l$ was very rare. right: $84.7 \%$, left: $75.0 \%$ ). There were 9 subjects (6.3\%: Types $d, d$-I,$d$-II, $e, f)$ in which Tin branched from $T t c$. There were 3 unusual cases $(2.1 \%)$ exhibiting Type 3- $a$ in which Tin branched independently from the 3rd part. Among 5 cases (3.5\%: Types $b$-I, $c, d$-II $)$ in which a lateral costal branch $(R c l)$ arose from Tin, there was a Type $1-d$-II subject (No. 352, 85-year-old male, left side) in which $\mathrm{Rcl}$ originated from Tin branching from a thyrocervical trunk (Ttc) consisting of the transverse cervical $(T c)$ and the suprascapular arteries $(S s)$. Moreover, in a Type 1-g subject (No. 174, 74-year-old female, right side), Tin originated from a common trunk consisting of $S s$ and a superficial branch $(R s)$ of $T c$.

4) $T t c$

As enormous variation exists in ramification of the thyrocervical trunk (Ttc), we based our classifi-

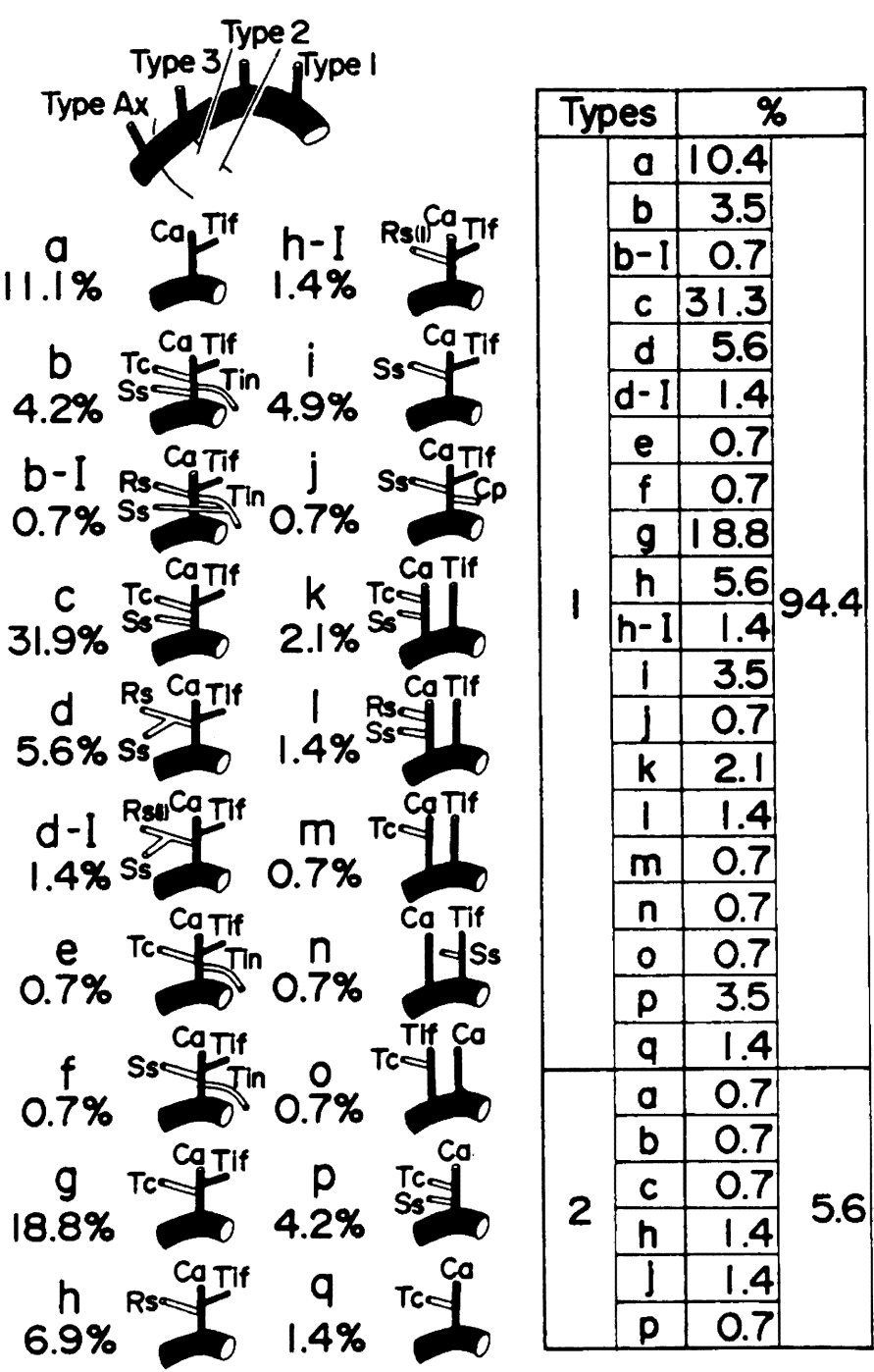

Fig. 5. Types of origin of the thyrocervical trunk (Ttc). The classification was mainly made by the common trunk of the ascending cervical $(\mathrm{Ca})$ and inferior thyroid arteries ( Tif) either existing or not. Type 1-c Ttc occurred most frequently, $31.1 \%$ of cases, and Typc a was in $11.1 \%$, in which its trunk arose alone from 1st (type 1-a: $10.4 \%$ ) or 2nd parts (type $2-a$ ) of the subclavian. 
cation mainly on the ascending cervical artery $(\mathrm{Ca})$, which supplies the spinal cord, and the inferior thyroid artery ( $T$ if $)$, which supplies the thyroid gland, as shown in Fig. 5. Ttc normally $(94.4 \%)$ branches in an ascending direction from the 1st part immediate medial to $s a$, but in some cases $(5.6 \%)$ it branched from the 2nd part immediately distal to the medial border of $s a$. The most common type observed in 45 cases $(31.3 \%$, males: $33.7 \%$, females: $27.6 \%$, right: $51.4 \%$, left: $11.1 \%$ ) was Type $1-c$ in which 4 branches ( $\mathrm{Ca}$, Tif, Ss, Tc) formed a common trunk branching from the 1st part. This type was observed with a higher incidence on the right side. This type included independent branching of $T c$ and $S s$ from $T t c$, and branching of both arteries as a common trunk from Ttc. Another type occurring with a high incidence of $18.8 \%$ was Type $1-g$ in which $S s$ was absent. There were 6 cases $(4.2 \%$, including Type $d$ of the $V e$ classification) in which Tif branched independently from $S u$ (Types $k, 1$ and $m$ ), and 8 cases $(5.6 \%)$ in which it was absent (Types $p, q)$. In 2 cases $(1.4 \%) \mathrm{Ca}$ arose independently from the one (Types 1- $n, 1-o$ ), no absent of $C a$ was observed. There were also 16 cases $(11.1 \%)$ in which a common trunk was formed by $\mathrm{Ca}$ and Tif alone (Type $a$ ). In 16 cases $(11.1 \%), C a$ and Tif did not form a common trunk (Types $k-q$ ). In the remaining cases $(88.9 \%)$, these two arteries formed a common trunk or they were combined with additional arteries to form the common trunk. As a definitive definition of what constitutes the thyrocervical trunk does not exist at present, for the purpose of this study we defined it as the last-mentioned type, which is either a common trunk consisting of $C a$ and Tif alone, or a common trunk consisting of $\mathrm{Ca}$ and Tif with another branch $(88.9 \%)$.

\section{5) $T c$}

Fig. 6 shows the data related to the transverse cervical artery $(T c)$ in the 144 arteries examined in this study. As described in the subject of Fig. 1, the final regions supplied by the superficial branch $(R s)$ are the upper and middle portions of the trapezius muscle after traversing the superficial layer of the levator scapulae muscle (Refer to $a \cdot b$ and $c \cdot d$ at the upper right of Fig. 6). This $R s$ sends a branch (upper portion branch) to the upper portion and a branch (middle portion branch) to the middle portion before penetrating the muscle. Type $e$ and Type $f$ are cases in which the upper portion branch and the middle portion branch are separate and originate from different sites. In this report, we have given the upper portion branch $(R s 1)$ the name of upper superficial branch, and the middle portion branch (Rs 2), the middle superficial branch.

There were 122 cases $(84.7 \%)$ in which $T c$ was present (Types $a, b, e$ ). Among these, there were 93 cases $(64.6 \%)$ in which $T c$ branched from Ttc (Type $a$ ). Of these 93 cases, $89(61.8 \%$, males: $66.3 \%$, females: $55.2 \%$, right: $58.3 \%$, left: $65.3 \%$ ) were Type 1- $a$ in which the branch originated from the 1st part. There were 22 cases $(15.3 \%)$ in which $R s$ and $R p$ branched independently (Types $c, d$, and $f)$. However, in Type $c(13.9 \%)$, Rs branched from Ttc (from the 1st part: $12.5 \%, 2$ nd part: $1.4 \%$ ), and $R p$ branched from the subclavian artery (from the 2nd part: $4.9 \%$, 3rd part: $8.3 \%, A x: 0.7 \%$ ). Furthermore, there was a subject (No. 174, 74-year-old female, right side) exhibiting Type 1.3- $d$ in which $R s$ and $R p$ branched from the 1st and 3rd parts of $S u$, respectively. Another extremely unusual subject (No. 321, 66-year-old female, left side) exhibited Type 1.2.3-f in which $R s(1)$ branched from $T t c$ (branch from the 1st part) and $R s(2)$ and $R p$ branched from the 2 nd and 3 rd parts of $S u$. The figure shows types in which $T c, R p$, and $R s$ branching directly from $S u$ are independent branches, and types in which they form a common trunk with other branches.

The figure at the bottom right of Fig. 6 shows the relation between $T c$ and the brachial plexus $(P l b)$. There were 104 cases $(72.2 \%)$ in which $T c$ traversed $P l b$ along an ascending course $(A)$, including Type $a$ (excluding one case in which branching was from the 2nd part), Type $b$ (Type 1-b) with branching from the 1 st part, and 4 cases $(2.8 \%$ ) of Type $c$ (Type 1.2-c) in which $R p$ branched from the 2 nd part. However, $T c$ of Types $a$ and $b$ only traversed the scalenus anterior muscle anteriorly. In the others, $R p$ or $T c$ traversed $B-F$ of $P l b$.

\section{6) Ss (Fig. 7)}

Type $a$, in which the suprascapular artery $(S s)$ branched independently appeared in 37 cases (25.7\%, males: $26.7 \%$, females: $24.1 \%$, right: $27.8 \%$, left: $23.6 \%$ ). In this type, $S s$ originated from the 2 nd part in $1.4 \%$, from the 3 rd part in $16.7 \%$, and from the axillary artery $(A x)$ in $7.6 \%$. The most commonly observed type was Type $b$ seen in 55 cases $(38.2 \%$, males: $39.5 \%$, females: $36.2 \%$, right: $37.5 \%$, left: $38.9 \%$ ), in which $T c$ and $S s$ (equivalent to Types $c, k$, and $p$ of the $T t c$ classification) branched from Ttc. The majority were Type $1-b$, in which branching was from the 1st part. This type was observed in 53 cases $(36.8 \%$, males: $37.2 \%$, females: $36.2 \%$, right: $37.5 \%$, left: $36.1 \%$ ). Type $g$ (equivalent to Types, $b$ and $b$-I of the Tin classification), in which $S s$ branched from Tin was observed in $4.2 \%$. There were 2 cases (No. 174. right and left sides) of Type $k$ in which two $S s$ were found. On both sides alike, $S s(1)$ from the 1st part traversed $s a$ anteriorly. then passed over $P l b$ (position $A$ in the center of the 


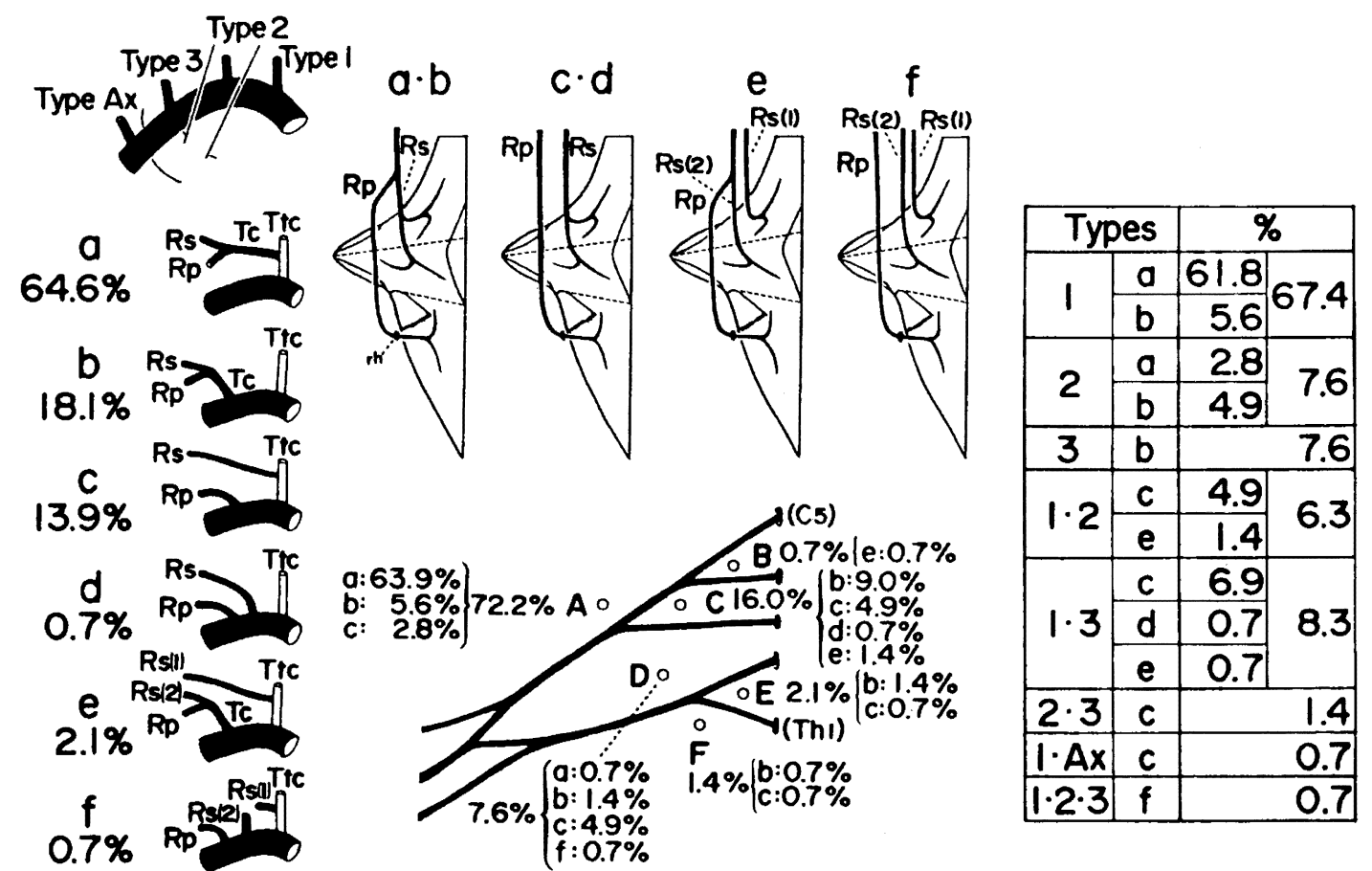

Fig. 6. Types of origin of the transverse cervical artery $(T c)$, and the frequency of the various positions of $T c$ as it passes through the brachial plexus. The left row shows some variations in the origin of $T c$. In Type $a(64.6 \%$ of cases) $T c$ arose directly from the thyrocervical trunk $(T t c)$, while in Type $c$ the superficial branch $(R s)$ and deep branch $(R p)$ of $T c$ originated separately from $T t c$ and the subclavian, respectively. Moreover, occasionally, the branch of $R s$ (named as the upper superficial branch $(R s(1)$ ) by authors) to the upper portion of trapezius muscle originated independently from $T t c$ and the branch $(R s(2))$ to its middle portion directly from either $R p$ or the subclavian (see Types $e$ and $f$, and the middle upper drawing showing the arteries supplying the muscle). Consequently, type 1.2.3. (the table on right side) intended to mean $R s(1), R s(2)$ and $R p$ arise from the 1 st, 2nd and 3 rd parts of the subclavian, respectively.

figure) and the superior scapular transverse ligament (Lss). Ss(2), which branched from the 3rd part and from $A x$, was transfixed to $P l b$ ( $C$ in the center of the figure), then passed under Lss to reach the supraspinatous fossa. In 4 cases $(2.8 \%), S s$ was absent.

The lowermost figure in Fig. 7 shows the relation between the form of $S s$ and $P l b$. Similar to $T c$, Ss that branched from the 1st part, usually passed over $P l b$ ( $A: 64.6 \%$ ) (excluding 1 case of Type $1-b$, and 1 case of Type 1-c). However, $S s$ branching from the 2nd part (2 cases of Type 2-a, 2 cases of Type $2-d, 1$ case of $2-e$, and 1 case of $2-g$ ) and $S s$ branching from the 3 rd part (1 case of 3-a) were found to traverse $A$ in a few cases. Those transfixed to $P l b(B$, $C$, and $D: 32.7 \%$ ) were largely $S s$ originating between the 2 nd part and $A x$, but a few exceptions were noted (Traversing $A: 2$ cases of Type 2-a, 2 cases of Type 2- $d, 1$ case of 2-e, 1 case of 2-g, 1 case of 3- $a$; transfixed to $P l b: 1$ case of Type 1-c, 1 case of Type $1-i)$.

\section{7) $T c c$ (Fig. 8)}

The costocervical trunk (Tcc) (Types $a-c$ ) was present in 96 cases $(66.7 \%)$, which is approximately $2 / 3$ rds of the total. In most cases, Tcc was independent and was Type $a$ originating from $S u$. Of the
Type $a$ cases $(65.3 \%$, males: $66.3 \%$, females: $63.8 \%$, right: $73.6 \%$, left: $56.9 \%$ ), Type $1-a$ in which branching was from the 1st part was observed in 33 cases $(22.9 \%$, males: $27.9 \%$, females: $15.5 \%$, right: $9.7 \%$, left: $36.1 \%$ ), and Type $2-a$ in which branching was from the 2nd part was observed in 61 cases (42.4\%, males: $38.4 \%$, females: $48.3 \%$, right: $63.9 \%$, left: $20.8 \%$ ). Type $1-a$ occurred mainly on the left side and Type 2-a on the right side. Separate branching (Types $d-i$ ) of the supreme intercostal artery $(I s)$ and the deep cervical artery $(C p)$ was found in 47 cases $(32.6 \%$, males: $32.6 \%$, females: $32.8 \%$, right: $25.0 \%$, left: $40.3 \%$ ). $1 s$ branched from a position proximal to $C p$ in all but one case of Type $g$. One case (Type $j$ ) was found in which $C p$ was absent.

8) Entire ramification of $\mathrm{Su}$

Fig. 9 shows our classification of the accurate branching sites and nature of common trunk formation of all the arteries arising from $S u$. We classified all of the arteries into three types (upper right of Fig. 9). Type I, which was characterized by the presence of Ttc formed from a common trunk consisting of $\mathrm{Ca}$ and Tif, was observed in $82.6 \%$ (119 cases). Type II, in which Ttc and Tin formed a common trunk, was seen in $6.3 \%$ (9 cases), and 


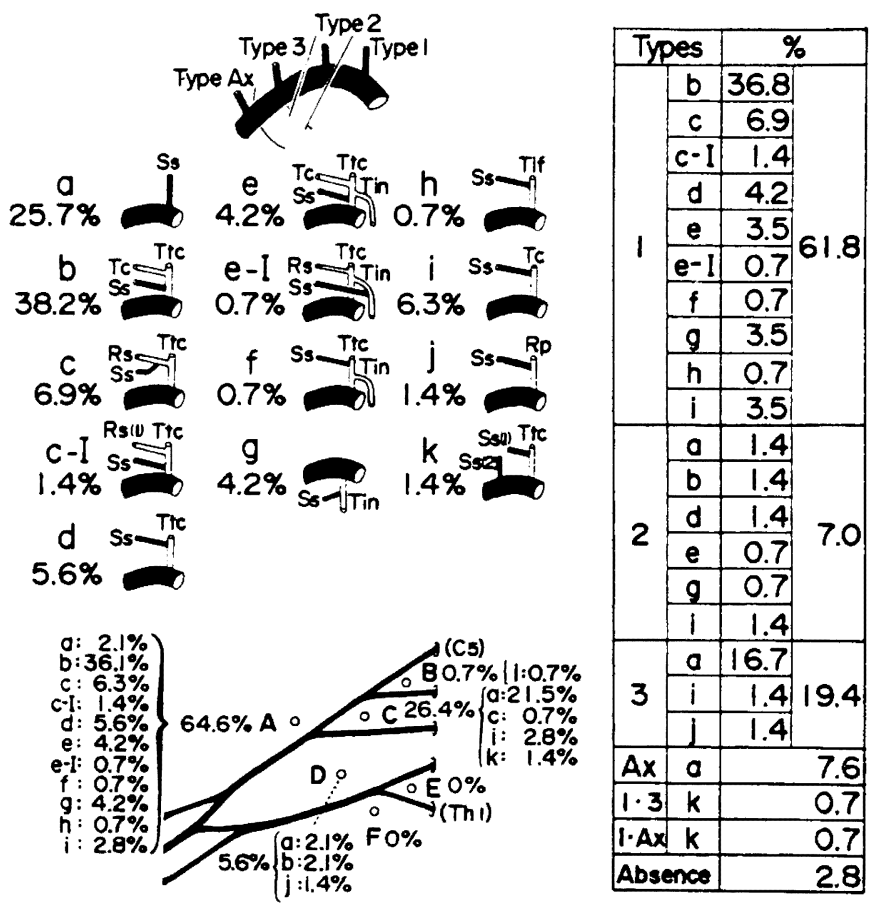

Fig. 7. Types of origin of the suprascapular artery $(S s)$, and the frequency of the various positions as it passes through the brachial plexus. The most frequent type was found to be Type $1-b$ in $36.8 \%$ of the cases in which the transverse cervical artery and $S s$ arose from the thyrocervical trunk as the common trunk or independent artery. In Type $k$, $1.4 \%$, two $S s$ existed. $S s(1)$ arose from $T t c$ which from the 1st part of subclavian, after passing over the brachial plexus it passed over the superior scapular transverse ligament; $S s(2)$ arose directly from either the 3rd part of (Type $1 \cdot 3-k$ ) or the axillary artery (Type $1 \cdot A x-k$ ), passing through the brachial plexus and under the ligament.
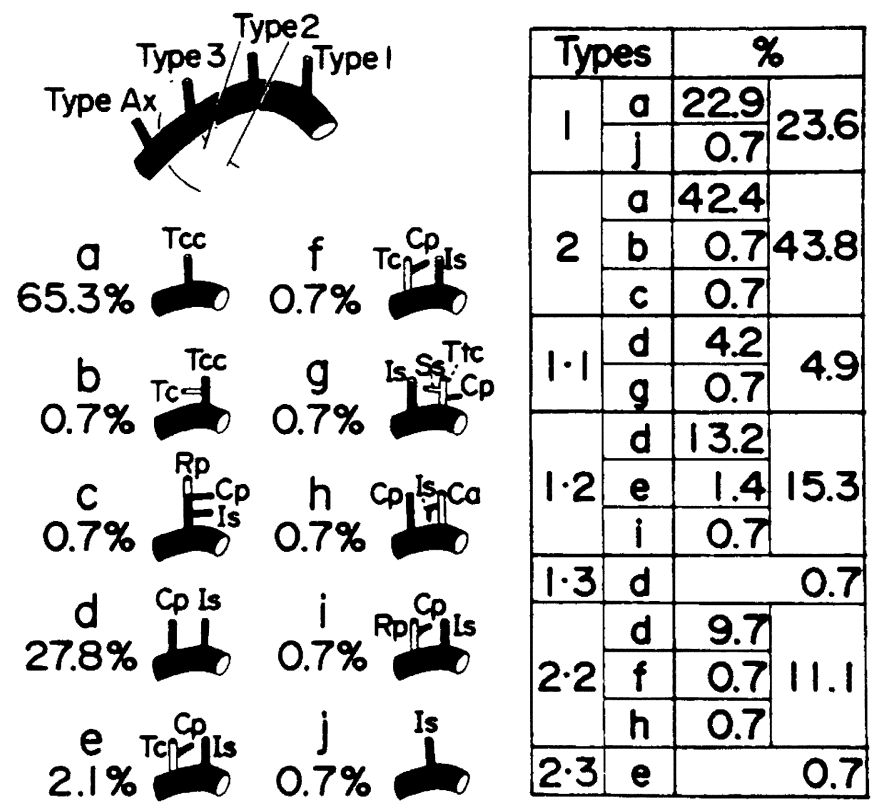

Fig. 8. Types of origin of the costocervical trunk ( $T c c)$. Although in Types $a \sim c(66.7 \%) T c c$ as the common trunk of the supreme intercostal artery(IS) and deep cervical artery $(C p)$ arose from the 1 st or 2 nd parts of the subclavian, in Types $d \sim i(32.6 \%)$ both arteries arose independently from the various positions of the subclavian, in which, except Type $g$ case, Is located more proximal to $C p$.
Type III, in which $\mathrm{Ca}$ and Tif branched independently or one of $\mathrm{Ca}$ or Tif was absent, was observed in $11.1 \%$ (16 cases). Since Type I appeared with the highest incidence, we classified it further as follows (Left of Fig. 9). In Type $A$, occurring in $31.9 \%, T c$ and $S s$ branched from Ttc (both branching independently from $T t c$, or branching from $T t c$ as a common trunk). In Type $B$, occurring in $18.8 \%$, only $T C$ branched from $T t c$. In Type $C$, occurring in $8.3 \%$, only a superficial branch $(R s)$ of $T c$ branched from $T t c$. In Type $D$, occurring in $6.9 \%, T c(R s)$ and $S s$ branched from $T t c$. In Type $E$, occurring in $5.6 \%$, only $S s$ branched from $T t c$, while in Type $F$, occurring in $11.1 \%$, only $T t c$ ( $C a$ and $T i f)$ was present.

Type I- $A(1)(13.2 \%$, males: $14.0 \%$, females: $12.1 \%$, right: $22.2 \%$, left: $4.2 \%$ ) exhibited the highest incidence among all of the types. In this type, Ve, Ttc and Tin branched from the 1st part of $\mathrm{Su}$, while $T c c$ branched from the 2nd part. Type I- $B(1)(9.0 \%$, males: $5.8 \%$, females: $13.8 \%$, right: $11.1 \%$, left: $6.9 \%$ ) exhibited the next highest incidence, with Ve, Ttc and Tin branching from the 1st part, $T c c$ from the 2nd part, and $S s$ from the 3rd part. This was followed by Type I- $A(2)(6.9 \%$, males: $8.1 \%$, females: $5.2 \%$, right: $1.4 \%$, left: $12.5 \%$ ), with $T c c$ branching from the 1st part, proximally from Tin in 5 cases, and distal from Tin in 5 cases. The 4th highest incidence was shown by Type I-C(1) $(5.6 \%$, males: $4.7 \%$, females: $6.9 \%$, right: $9.7 \%$, left: $1.4 \%$ ), with Ve, Ttc and Tin branching from the 1st part, $T c c$ from the 2nd part, and $T c(R p)$ and $S s$ from the 3rd part.

In these four most frequently occurring types, $T c c$ branched from the 2 nd part on the right side and from the 1st part on the left side in the majority of cases, indicating the existence of a sides difference.

\section{Discussion}

The subclavian artery $(\mathrm{Su})$ traverses the interscalene triangle dorsal to the scalenus anterior muscle (Type I of Fig. 2: 98.6\%), is located ventral to the brachial plexus $(P l b)$ in the interscalene triangle, traverses the scalenus anterior muscle anteriorly (Type III: $0.7 \%$ ), or is transfixed to the scalenus anterior muscle (Type II: $0.7 \%$ ). Type III has been reported in 4 white subjects $\left.(1.1 \%)^{11.27}\right), 2$ negro subjects $(0.3 \%)^{12)}, \quad 2$ adult Japanese subjects $(0.6 \%)^{1)}$, and $2 \mathrm{Japanese}$ fetuses $(1.7 \%)^{28)}$. Including our cases, a total of $11 / 1738(0.6 \%)$ have been found, with a slightly higher incidence on the left side. Type II has been reported in 1 Japanese subject $(1.0 \%)^{38)}, 7 \mathrm{Japanese}$ fetuses $(6.0 \%)^{28)}$, and African negro subjects with a frequency of $17.2 \%^{19}$, thus showing a significant racial difference. Although the 

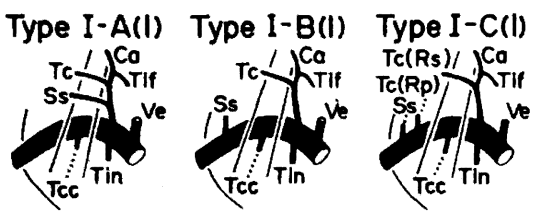

Type I-A(2)

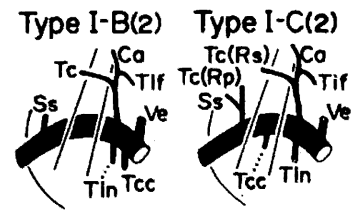

Type I-A(3)
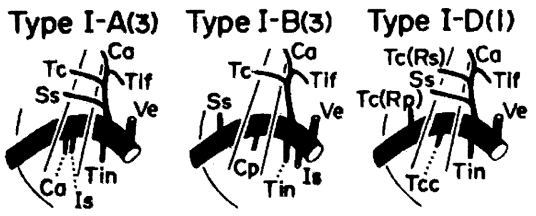

Type I-A(4)
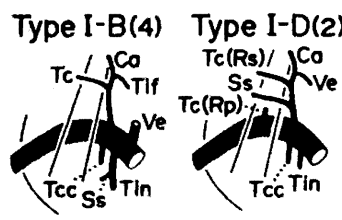

Type I-A(5)
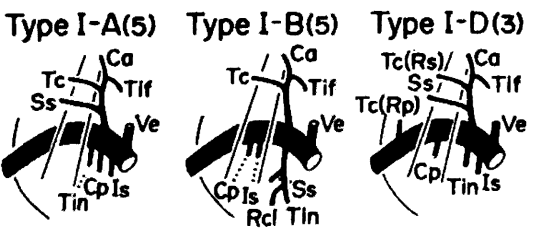
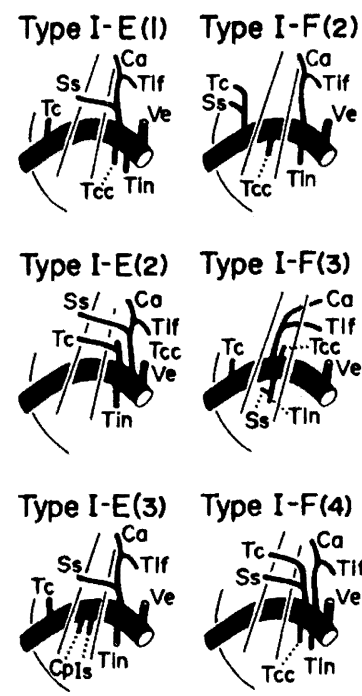

Type I-F(4)
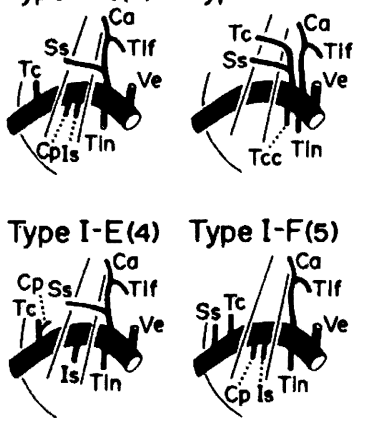

Type I-F(5)
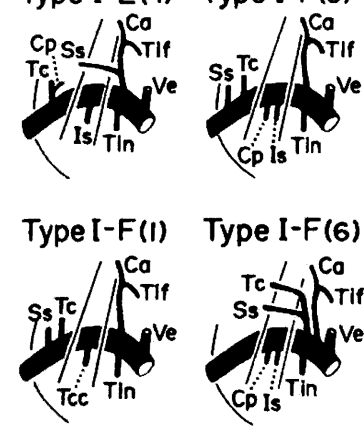

Type I-F(6)

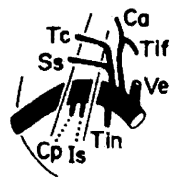

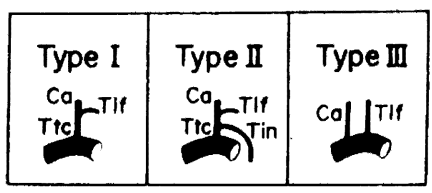

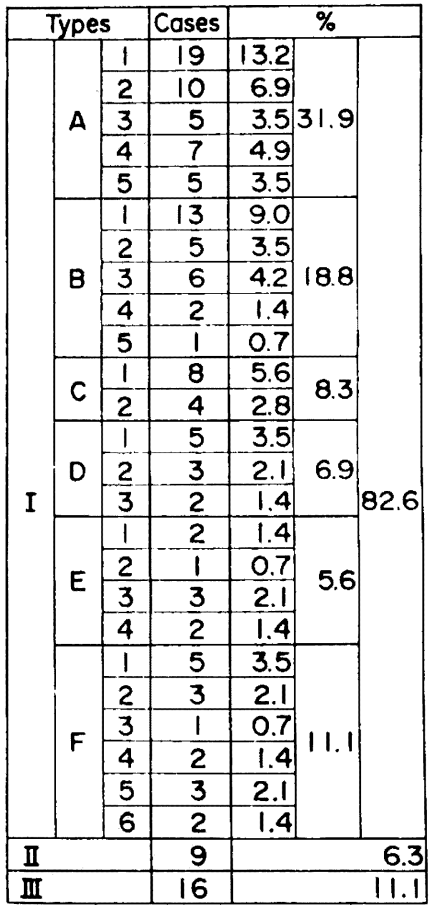

Fig. 9. Types of origins of the whole branches from the subclavian artery. Type I has the thyrocervical trunk (Ttc) formed by the ascending cervical $(\mathrm{Ca})$ and inferior thyroid arteries (Tif), Type II Ttc with the internal thoracic artery (Tin), and Type III $\mathrm{Ca}$ and Tif as independent arteries from the subclavian. Further, Type I was totally subdivided into 25 types. The most commonly appeared type was found to be Type I-A(1) in $13.2 \%$ of cases, in which Ve, Tin, and Ttc with $T c$ and $S s$ arose from the 1 st part, and $T c c$ from 2 nd part of the subclavian.

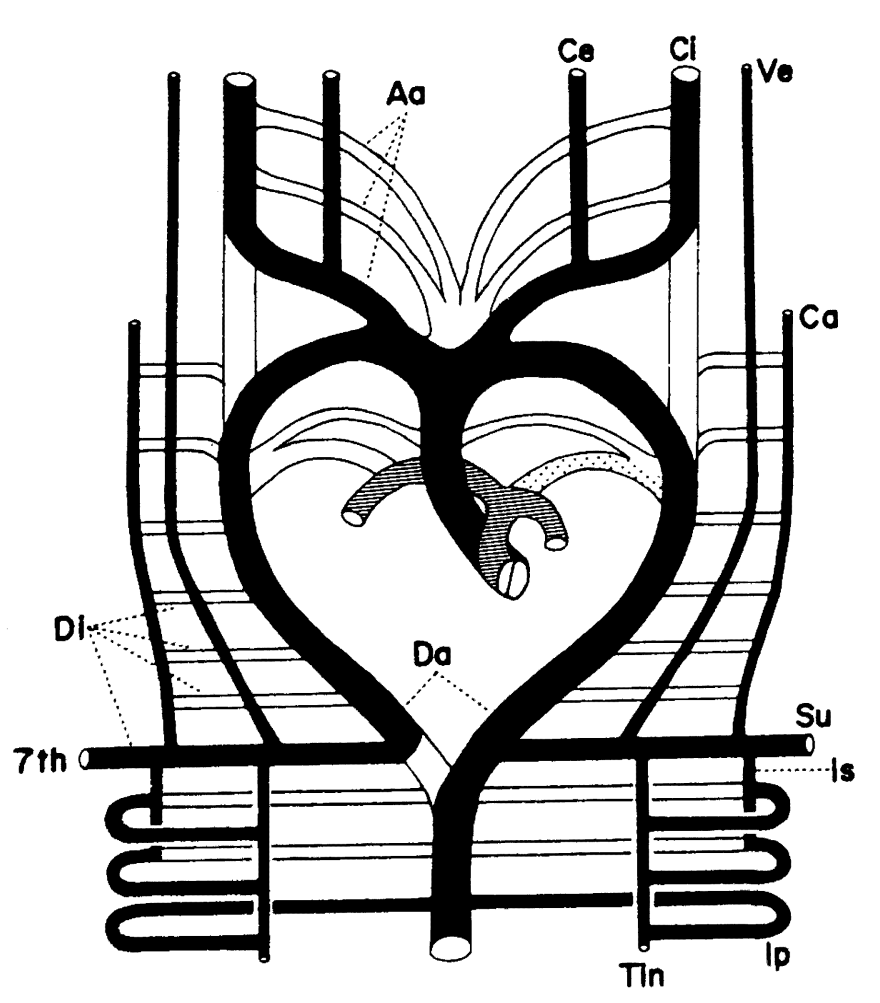

positional relation between the subclavian artery and the scalenus anterior muscle is of great importance clinically, the mechanism of formation of these types has remained unclear. We conducted this study in an attempt to provide an explanation.

Normally, the scalenus muscles are classified into the scalenus anterior, medius and posterior muscles, but this classification is only based by convenience on the position of the terminal portion. The scalenus anterior muscle $(s a)$ and the scalenus medius muscle $(\mathrm{sm})$ arise from the anterior and posterior tubercles, respectively, of the transverse process of the cervical vertebra. The subclavian artery is located anteriorly, and the brachial plexus posteriorly between $s a$ and $s m$. The scalenus minimus muscle $(s m i)$ is a small muscle bundle appearing between $s a$ and $s m$. However, it is regarded as a muscle bundle that moves posteriorly from $s a$, because it originates from the anterior tubercle of the transverse process of the 6th

Fig. 10. Schematic diagram, showing the various components of the aortic arch complex and their transformations in the early stage of human embryo, to explain the variations in origins of the branches from the subclavian artery. It was modified from Arey's (65). 
or 7 th cervical vertebra, runs inferolaterally, then terminates above the 1 st rib between the subclavian artery and the brachial plexus. ${ }^{39)}$ The incidences of smi appearance reported by Stott $^{41)}(39 / 100$ sides: $39.0 \%)$, Boyd $^{8)}(10 / 30$ sides: $33.3 \%)$ and Serizawa ${ }^{38)}$ $(32 / 100$ sides: $32 \%)$, are in close agreement, suggesting that overall, it appears somewhat more frequently on the right side (44/81 sides).

Serizawa reported finding 2 cases $(2 \%)$ in which a portion of the muscle bundle at the lower posterior extremity of the scalenus anterior muscle had separated and ran a posterior course terminating in the superior wall of the subclavian artery. He also reported 1 case (Type II) in which a similar muscle bundle passed over the superior wall of the subclavian artery, turned posteriorly, then terminated on the 1 st rib. In other words, in this case, the subclavian artery was transfixed to the scalenus anterior muscle. The former cases are of considerable interest as they appear to constitute a type intermediate between Type I (subclavian artery runs posterior to the scalenus anterior muscle) and Type II (the subclavian artery is transfixed to the scalenus anterior muscle).

Ontogenetically, the upper limb buds can be seen from the surface in the middle of the 4 th week of the embryonic stage, and at the beginning of the 5th week, the brachial plexus and the main stem of the subclavian artery have already penetrated them. ${ }^{5,10,26)}$ In this period, precartilage of the ribs can be seen. ${ }^{26)}$ A little later, muscle in the cervical region is formed, and the premuscle-mass that appears in the shoulder girdle differentiates, so that the form and positions of the adult muscles are largely determined by the end of the 7 th week. ${ }^{5)}$ On the basis of the above-described reports, it appears that the three types of positional relation between the subclavian artery and the scalenus anterior muscle are formed between the 5th and 7th weeks of embryonic development.

Accordingly, we considered that the primordium of the scalenus anterior muscle which envelopes the subclavian artery anteroposteriorly that named Type II should be the arche type. During the course of ontogenesis, the posteriorly orientated muscle bundle normally disappears (Type I), but in some rare cases, the anteriorly orientated muscle bundle disappears and the posterior muscle bundle remains, giving rise to Type III. Moreover, cases in which a portion of the muscle bundles of the scalenus anterior muscle terminate in the superior wall of the subclavian artery are transitional type of Type I and II cases. Muscle bundles orientated posteriorly in Type II do not separate from the scalenus anterior muscle bundles, but their site of origin is shifted slightly posterior, and those arising directly from the anterior tubercles of the cervical vertebrae, we propose constitute the scalenus minimus muscle.

Classification of the entire ramification from $\mathrm{Su}$ :

There have been numerous reports on the sites and morphology of ramification of branches from the subclavian artery $(S u)$, but in all cases, descriptions have been limited mainly to only a portion of the arteries $\left.{ }^{14}, 17,18,21,22,33-35,44\right)$ and to variation $9,16,20,25,36,47)$. Hence, the sites of origin of many of the arteries have remained unclear. ${ }^{27,29,33,35)}$ De Garis, ${ }^{13)}$ Daseler et al., ${ }^{12}$ ) Nizankowski et al. ${ }^{29)}$ and Adachi ${ }^{1)}$ gave detailed descriptions of each of the arteries branching from $S u$ in American, Polish and Japanese subjects, but they did not report the sites of origin of some of the arteries, nor classify the entire $S u$. Therefore, despite the fact that the incidence of each artery was definitively established by these reports, an overall picture of the combination of these $S u$ branches was not provided. Consequently, the most commonly occurring type of overall $S u$ morphology and its incidence has remained unclear.

We summarized the entire array of arteries arising from $S u$, as shown in Fig. 9 and found that among Japanese adult subjects of this study, Type I, in which the common trunk formed by the ascending cervical artery $(\mathrm{Ca})$ and the inferior thyroid artery (Tif) contains branches other than the internal thoracic artery (Tin), appeared with the highest frequency $(82.6 \%)$. Furthermore, the most commonly appearing forms of Type I were Type I - $A(1)$ with an incidence of $13.2 \%$, Type I $-B(1)$ with an incidence of $9.0 \%$, and Type I - $A(2)$ with an incidence of $6.9 \%$. Although differences in the classification conditions preclude from a direct comparison, Bean reported finding (in white and negro subjects) an incidence of $30 \%$ for a form of Type $I-D(2)$ in which the costocervical trunk $(T c c)$ branched from the 2 nd part, an incidence of $27 \%$ for Type I $-A(1)$, and $22 \%$ for Type I $-C(1)$. Daseler et al. found in their classification of branches excluding $\mathrm{Ca}$ and $T c c$ (in white and negro subjects), incidences of $46.8 \%$ for a type equivalent to Type I - $D(1)$ (our study: $3.5 \%$ ) and $25.3 \%$ for Type $\mathrm{I}-A(1)$, indicating a substantial difference among races. Moreover, among the four types (Types $A(1), A(2), B(1), B(2)$ ), the incidence of Type (1) in which Tcc arises from the 2 nd part on the right side was $75.0 \%(24 / 32$ sides), while the incidence of Type (2) in which $T c c$ arises from the 1st part on the left side was $86.7 \%$ (13/15 sides), showing a marked sides difference.

From these results, it can be seen that the morphology of branch ramification from the subclavian artery in our study of Japanese adults was characterized by exceptional variation.

The following is the findings of each artery. 
$V e$ :

The incidence of Types 1-a, $b$ and $c$ (Fig. 3), in which the vertebral artery $(V e)$ arose from the 1 st part of $S u$ independently, was highest at $94.5 \%$. Difference in the incidence of this type has not been found among the white, ${ }^{13,29)}$ negro, ${ }^{13)}$ and Asian, ${ }^{27,28)}$ races. A trend appears to exist for a slightly higher incidence among Asian races of Type $A$ (individual incidence of $6.9 \%$ ), in which the left $\mathrm{Ve}$ branches from the aortic arch, $7,9,13,27-29)$ but a difference has not been found between the fetus and adult. There have also been reports of $1.4 \%$ incidence of a common trunk with Tif (Type $d),{ }^{7,29)}$ and rare cases in which Tif traverses Ve posteriorly.

Tin:

Type $a$, in which the internal thoracic artery (Tin) arises independently from $S u$ have appeared with the highest incidence $(87.5 \%)$, almost without variation between white $(88.9 \%: 446 / 524),{ }^{13,22,29)}$ Asian $\left.(87.0 \%: 374 / 430),{ }^{1,27,}{ }^{*}\right)$ and negro races $(83.9 \%$ : $146 / 174)$. Furthermore, almost identical incidences of Type I $-a(79.9 \%)$ have been reported by Bean $(80 \%)$ and Daseler et al. (79.2\%). Occasionally, the $\mathrm{R}$. costalis lateralis $(R c l)$ has appeared in a descending course along an almost midaxillary line of the lateral wall of the thoracic cavity. This $\mathrm{Rcl}$ most commonly appeared as Type $c(1.4 \%)$ which branches from Tin. Type $c$ appeared with an incidence of $4.8 \%$ whites, ${ }^{13)} 9.8 \%$ in negroes,${ }^{13)} 17.9 \%$ in whites and negroes combined, ${ }^{7)} 11.9 \%$ in Asian adults, ${ }^{1,27)}$ and $19.8 \%$ in Asian fetuses, ${ }^{28,47)}$ indicating a slightly lower incidence in whites. In our study, we observed some rare cases of a type of $R c l$ (Type $d-\mathrm{II}: 0.7 \%$ ) originating as a branch of Tin which had arisen from Ttc, and another type (Type $b$-I: $1.4 \%$ ) originating from Tin which formed a common trunk with Ss. (The star-like symbol indicates the data from our study)

Ttc:

According to the report of Thomson $(1891),{ }^{44)}$ the thyrocervical trunk ( $T t c)$ or thyroid axis which he described is our Type $c(31.9 \%)$ formed as a common trunk by the inferior thyroid artery (Tif), the ascending cervical artery $(\mathrm{Ca})$, the suprascapular artery $(S s)$ and the transverse cervical artery (Tc). The incidence of this Type $c$ has been reported variously to be $33.7 \%(326 / 968)$ in white adults, ${ }^{13,29,44)} 15.0 \%(30 / 200)$ in white fetuses, ${ }^{29)}$ $32.2 \%(56 / 174)$ in negroes, ${ }^{13)} 27.3 \%(245 / 899)$ in whites and negroes combined, ${ }^{7,12)} 38.6 \%(130 / 337)$ in Asian adults, $\left., 17,{ }^{*}\right)$ and $33.3 \%(38 / 114)$ in Asian fetuses ${ }^{28)}$ indicating approximately equal incidences among different races. Among whites populations, higher incidences have been found in British $(44.7 \%),{ }^{44)}$ and American $(51.0 \%)^{13)}$ people, and a particularly low incidence in Polish people
$(15.0 \%) .^{29)}$ The incidences of Type $d$, which exhibited a common trunk formed by $C a$, Tif, Ss and a superficial branch of the transverse cervical artery, have been reported variously to be $45.2 \%$ (524/ $1160)$ in white adults, ${ }^{13,29,35,44)} 67.0 \%(134 / 200)$ in white fetuses, ${ }^{29)} 29.3 \%$ (51/174) in negroes, ${ }^{13)} 46.8 \%$ $(360 / 770)$ in whites and negroes combined, ${ }^{12)} 11.9 \%$ $(40 / 337)$ in Asian adults, ${ }^{\left.1,27,{ }^{*}\right)}$ and $11.4 \%(13 / 114)$ in Asian fetuses. ${ }^{28)}$ These results show that this Type $d$, appearing in more than half of the total, is remarkably common among whites, whereas it only appears in approximately $10 \%$ of Asians, including fetuses. The incidence of Type $g$, which is a form of Type $c$ lacking $S s$, has been reported to be only $0-4 \%$ in whites and negroes, $7,12,13,29,35,44)$ in contrast to the finding of $20 \%$ in Asians, $\left.{ }^{27,}{ }^{*}\right)$ indicating that it is a type appearing more commonly in Asians. In our study, we found that Type $a$, which exhibits a common trunk formed by $C a$ and Tif only, appeared with an incidence of $11.1 \%$, but has been little reported by other researchers. Reports of Type $a$ have only been made by Röhlich, ${ }^{35}$ ) who observed it in $4.2 \%$ (Hungarians), Adachi, who obtained an incidence of $1.7 \%$ (Japanese), and Mori who obtained an incidence of $1.8 \%$ (Japanese fetuses).

The thyrocervical artery is formed from a common trunk consisting of the above-mentioned four arteries. However, as shown in Fig. 5, there are numerous combinations of their branches, which means it is not clear exactly how many arteries are combined to form the thyrocervical artery, and a consistent definition by different researchers has not yet been obtained.

Ontogenetically, $\mathrm{Ca}$, which supplies the spinal cord, consists of residual dorsal intersegmental arteries that supply the neural tube segmentally and a portion of their longitudinal anastomotic channels, and appears at the earliest period. This is followed by Tif, which is observed from the 3rd to the 7 th week of embryonic development ${ }^{2,6,15)}$ until the primordium of the thyroid gland is formed. By the beginning of the 7 th week, $T c$ and $S s$ have extended to a premuscle-mass in the periphery of the scapula including the trapezius muscle, which has a form almost identical to that of the adult. ${ }^{5,26)}$

On the basis of this and consideration of the derivation of the name, thyrocervical trunk, we defined the prototype of Ttc as a common trunk consisting only of $C a$ and Tif (Type $a$ ). We propose that all of the combinations of other arteries with this fundamental arterial trunk be termed the thyrocervical trunk (Type $a-j: 88.9 \%$ ), and forms in which Tif and $\mathrm{Ca}$ arise independently, or either one is absent (Type $k-q: 11.1 \%$ ) be excluded from the definition of thyrocervical artery. 
$T c$ :

The transverse cervical artery $(T c)$ refers to a common trunk formed from a superficial branch $(R s)$ and a deep branch $(R p)$. In our classification (Fig. 6), it is equivalent to Types $a$ and $b$. Usually, $R s$ is an artery that exhibits an extremely stable course, arising from $S u$ then running posterolaterally and passing around the lateral border of the levator scapulae muscle to reach a superficial layer. During this course, it sends small branches and supplies the upper and middle portions of the trapezius muscle. According to the report of our previous study ${ }^{43}$, immediately before $R s$ penetrates this muscle, it divides to send a superior branch to the upper portion of the trapezius muscle (clavicular portion) and a middle branch to the middle portion (acromial portion) (Types $C, D: 84.1 \%$ ), and in some cases the superior branch may bifurcate at a site proximal to the site of $R s$ and $R p$ branching (Type $B: 11.4 \%$ ), or in rare cases, may branch independently from $T t c$ (Type $A: 4.5 \%$ ), or $R s(1)$ forms as a superior branch that arises directly and independently from Ttc. We named this $R s(1)$, the upper superficial branch. It has been reported appearing in rare cases by Thomson (Type $E \& P^{2}: 4.6 \%$ ), De Garis Type $E$ $\& P^{2}: 1.0 \%$ [whites], 5.2\% [negroes]), and by Yamada. ${ }^{46)}$ The type in which this artery appeared is equivalent to our Type $e(2.1 \%)$. After branching from $S u, R p$ follows a posterior course around the lateral border of the levator scapulae muscle to reach the deep layer of that muscle. It follows the border between the ventral sides of the rhomboid and serratus anterior muscles along a descending course to supply both muscles. During this course, one branch is transfixed to the rhomboid muscles, becoming a branch that supplies the lower portion of the trapezius muscle. This artery follows a highly defined course that allows easy identification of the artery. In extremely rare cases, this $R p$ branches from, the 3rd part of $S u$ or the 1st part of $A x$, then is transfixed to a muscle bundle of the serratus anterior at the 1st intercostal space, runs backward on the dorsal surface of this muscle to reach the medial border of the scapula. From there it follows the above-described course (unpublished data).

From the above-described findings, it appears likely that phylogenetically, $R s$ and $R p$ constitute a different system of arteries as they are separated by a group of the intermediate external oblique muscles (levator scapulae, rhomboideus major and minor, serratus anterior muscles) ${ }^{43)}$, with $R s$ following a course to the superficial layer and $R p$ to the deep layer of these muscles.

The most common among our cases was Type $a(64.6 \%)$, most of which were Type 1-a $(61.8 \%)$ with branching from the 1st part. The incidence of this Type $a$ has been reported variously to be $35.8 \%(495 / 1382)$ in white adults, $\left.{ }^{13}, 21,29,33,34,44\right)$ $15.0 \%(30 / 200)$ in white fetuses ${ }^{29}{ }^{29} 37.9 \%(66 / 174)$ in negroes, ${ }^{13)} 33.6 \%(355 / 1057)$ in whites and negroes combined. $\left.{ }^{7}, 12,17,18\right) 61.0 \%(308 / 505)$ in Asian adults, ${ }^{1,27,30, *)}$ and $57.9 \%(66 / 114)$ in Asian fetuses ${ }^{28)}$ indicating that this type is particularly common among Asians. The incidence of Type $c$, in which $R s$ arises directly from $T t c$ and $R p$ from $S u$ (including a common trunk of $R p$ and other branches), has been reported to be $37.2 \%(241 / 648)$ in whites, ${ }^{13,44)} 47.1 \%(82 / 174)$ in negroes, ${ }^{13)} 61.5 \%$ $(571 / 928)$ in whites and negroes combined, ${ }^{12,17,18)}$ $17.6 \%(56 / 319)$ in Asian adults, $\left.{ }^{27,} 30,{ }^{*}\right)$ and $27.2 \%$ $(31 / 114)$ in Asian fetuses, ${ }^{28)}$ indicating that this Type $c$ is particularly common among whites and negroes (appearing in more than half) and less common among Asians (appearing in approximately $20 \%$ ). The incidence of Type $b$, which was relatively high among Japanese subjects $\left.(16.2 \%: 40 / 247),{ }^{30,}{ }^{*}\right)$ has been reported to be $7.7 \%(50 / 648)$ in whites, ${ }^{13,44)}$ $6.3 \%$ in negroes, ${ }^{13)} 20.8 \%$ in whites and negroes combined, ${ }^{17)}$ and $4.2 \%$ in Chinese. ${ }^{27)}$ Therefore, with the exception of the report of Huelke, ${ }^{17)}$ the incidence of Type $b$ has been low. We also found some extremely rare cases who exhibited Types $d-f$, which have been little reported in the past. From the above-described findings, it can be seen that in the early stage of development when $T c$ is formed from the primary capillary network, there are three arteries feeding the target organs of the upper, middle, and lower portions of the trapezius muscle, each branching independently from $S u$. We consider this configuration to be the prototype (equivalent to Type $f$ of Fig. 6) of the variations described below. We believe that $R s(1)$ forms a common trunk with Ttc. $R s(1)$, which branches from the 1st part of $S u$ positioned somewhat cephalically, and $R s(2)$ traverse $s a$ ventrally, and pass over $P l b$ (because $P l b$ is positioned caudally to these arteries). $R p$, which arises from the 3 rd part positioned somewhat caudally to these, is transfixed to $P l b$ ( $P l b$ is located at the course of this artery), then follows a course posteromedial to the superior angle of the scapula. These arteries, similar to those supplying the gastrointestinal tract, are considered in principle, to supply their target organs via the shortest and most direct course possible at this stage of development. We believe that the positional relationships between $\mathrm{Plb}$ and these arteries are already established at this stage and retained through to the adult stage, a proposal supported by similar findings reported by other researchers. ${ }^{1,21,28)}$ These findings indicate that the site of bifurcation of these arteries from $S u$ is the most important factor in determining the position of their course in relation to $\mathrm{Plb}$. Furthermore, we 
suggest that communicating anastomotic branches between these three arteries exist dorsally beyond $P l b$, and that the site and branching form of origin of $T c$ is determined by differences in the sites of disappeared origin of these three arteries, and the site of disappearance of these communicating branches. In other words, we propose that the sites of origin of $R s(2)$ and $R p$ have disappeared in Type $a$, and the site of origin of $R s(2)$ and the communicating anastomotic branches between $R p$ and $R s(2)$ have disappeared in Type $c$.

Diverse opinion exists among researchers regarding the definition of the term used as transverse cervical artery. In Type $c$, which appears with a high incidence among whites and negroes, $R s$ and $R p$ arise independently, which means that $T c$ does not exist as a common trunk formed both of these arteries, hence various names have been given to these two arteries. Numerous researchers have termed $R s$ as the superficial cervical artery, $1,12,13,28,29,34,44,46)$ ascending ramus, ${ }^{7)}$ ascending branch, ${ }^{13)}$ transverse cervical artery, ${ }^{17,18)}$ and superficial transverse cervical artery, ${ }^{14,23)}$ and $R p$ as the transverse cervical artery, ${ }^{1,12,13,29,34)}$ descending ramus, ${ }^{7)}$ descending branch, ${ }^{13)}$ posterior scapular artery, ${ }^{7,14,44)}$ dorsal scapular artery, ${ }^{17,18)}$ and descending scapular artery. ${ }^{46)}$ Furthermore, the independent $R s$ branch to the upper portion of the trapezius muscle, which we termed the upper superficial branch, has been described as the A. cervicalis superficialis accessoria $^{46)}$ and the superficial cervical artery, ${ }^{13,44)}$ similar to the name used for $R s$. Various researchers have been using the same name (transverse cervical artery, etc.) to describe different arteries, and several names to describe the same artery, which has led to confusion in the description of this artery. For this reason, an attempt should be made at the very least, to identify each of the above-described three arteries after following their course to the region where they finally terminate, in order to ensure the consistent use of anatomical terms.

Ss:

The most common form of suprascapular artery $(S s)$ observed in our study was Type $b$ (Fig. 7). The incidence of this type has been reported variously to be $33.5 \%(324 / 968)$ in white adults ${ }^{13,29,44)} 15.0 \%$ $(30 / 200)$ in white fetuses, ${ }^{29)} 29.9 \%(52 / 174)$ in negroes, ${ }^{13)} 19.7 \%(178 / 904)$ in whites and negroes combined, ${ }^{7,12)}$ and $53.7 \%(190 / 354)$ in Asians, ${ }^{\left.1,27,{ }^{*}\right)}$ indicating a high frequency of this type among Asians. Type $c$, which appeared with a low incidence in our study, has been reported by others to have an incidence of $52.8 \%(603 / 1142)$ in white adults, ${ }^{13,29,35,44)} 67.0 \%(134 / 200)$ in white fetuses, ${ }^{29)}$ $43.7 \%(76 / 174)$ in negroes, ${ }^{13)} 59.4 \%(460 / 775)$ in white and negroes combined, ${ }^{12)}$ and $8.8 \%(19 / 216)$ in Asians, $\left.{ }^{27},{ }^{*}\right)$ thus showing a high incidence in whites and negroes. The incidence of Type $a$ branching independently from $S u$, a type observed in approximately $1 / 4$ of our subjects, has been reported to be $6.0 \%(63 / 1048)$ in white adults $\left.{ }^{13}, 29,33,44\right)$ $2.5 \%(5 / 200)$ in white fetuses, ${ }^{29)} 14.5 \%(59 / 406)$ in negroes, $\left.{ }^{13}, 33\right) 16.6 \%(150 / 904)$ in whites and negroes combined, ${ }^{7,12)} 21.2 \%(75 / 354)$ in Asian adults, $\left.{ }^{1,}, 27,{ }^{*}\right)$ and $16.7 \%(19 / 114)$ in Asian fetuses, ${ }^{28)}$ thus showing an incidence in the decreasing order of Asians, negroes, and whites. The extremely rare Type $k$, in which there are two $S s$, was found in whites by Thomson $(1.5 \%)$, in negroes by De Garis $(2.9 \%)$, and in the Japanese by Adachi (1.4\%), the authors $(1.4 \%)$, and Mori (fetuses: $3.5 \%$ ), indicating the absence of any racial difference in the distribution of this type.

Although there were some exceptions, similar to the case with $T c$, in general the relation between $S s$ and the brachil plexus was as follows. The artery arising from the 1st part passed over the plexus ( $A$ in Fig. 7), then followed a course over the superior scapular transverse ligament. In contrast, the artery arising from the 2nd or 3rd part was transfixed $(B-E)$ to the nerve plexus, and followed a course under the ligament. As similar findings were reported by Adachi and Mori, whether or not this artery passes over or is transfixed to the brachial plexus is determined by the position at which $S s$ branches from $S u$, similar to the case with $T c$. Furthermore, it can be surmised from the above that in the early period when $S s$ arises, two arteries exist as in Type $k$, but one of these disappears during development (normally, the more distal of the two arteries disappears).

Tcc:

The costocervical trunk (Tcc) is formed by a common trunk composed of the supreme intercostal artery $(I s)$ and the deep cervical artery $(C p)$, and is equivalent to our Type $a$. The incidence of Type $a$ has been reported variously to be $72.2 \%(231 / 320)$ in white adults, ${ }^{29)} 73.5 \%(147 / 200)$ in white fetuses, ${ }^{29)} 89.1 \%(907 / 1018)$ in white and negroes combined, ${ }^{7,12,13)} 73.6 \%(159 / 216)$ in Asian adults, ${ }^{\left.1,{ }^{*}\right)}$ and $91.2 \%(104 / 114)$ in Asian fetuses, ${ }^{28)}$ indicating an absence of any substantial racial difference. De Garis and Daseler reported finding almost no difference in the incidence of Types 1- $a$ and 2-a among Type $a$ cases (each was approximately $45 \%$ ), but our study revealed a higher incidence of Type $2-a(42.9 \%)$ than that of Type 1-a $(22.9 \%)$, and a higher incidence of Type 2- $a$ on the right side and a higher incidence of Type 1- $a$ on the left. In Types $d-i$, in which $C p$ and $I a$ branch independently, $I s$ branched from a more proximal position than did $C p$, except for one case of Type $g$. Since a similar 
finding has been reported in a $12-\mathrm{mm}$ pig embryo, ${ }^{45}$ ) it appears likely that in the early embryonic stage of man, who is also a species of mammal, $I s$ and $C P$ branch independently (with a higher probability of $I s$ being in a more proximal position than $C p$ ), with a communicating branch between the two. During the course of development, Type $d$ arises as a result of the communicating branch disappearing, a form observed in $5-10 \%$ in all of the races studied. ${ }^{1,12,13)}$ The remainder manifest as Type $a$, which arises by the site of origin of one of the two arteries disappearing. In our study, we found a fairly high incidence of Type $d$.

Ontogenetic Considerations:

As can be seen from the above, there is extreme variation in the sites of origin of the arteries branching from $S u$. The reason for this diversity may be that $S u$ is formed through a complex development process, and that it traverses the shoulder joint, which is the most kinetic within the body.

Ontogenetically, the right and left dorsal aortae fuse $^{6)}$ caudally from the 9 th segment at the end of the 4 th week of development (4 mm embryo), $\left.{ }^{10}\right)$ resulting in a morphology as shown in Fig. 10. In this period, dorsal intersegmental arteries extend from the dorsal aortae towards the dorsally sited neural tube. Their main branches supply the neural tube, while a portion of branches are oriented ventrally along the body wall. There is a few divergent opinion $^{2,10)}$, but in humans, the 7 th dorsal intersegmental artery from the cephalic end of the embryo develops into $\mathrm{Su}^{6,15)}$ At the beginning of 7 th week (days $44-48)^{6,10)}$ the white section (the portion where the right and left sides are fused) of the right dorsal aorta shown in Fig. 10, gradually narrows and disappears. $S u$ shifts cephalically with the descent of the heart which began in the 2 nd month. As a result, the derivation of $S u$ on the left and right sides is different, with the left $S u$ forming from the 7th dorsal intersegmental artery alone, and the right $S u$ forming from the right 4 th aortic arch, the dorsal aorta of the 3rd-7th segments, and the 7th dorsal intersegmental artery. This developmental difference may be a cause of differences in the right and left sides.

Details of the period up to the 7th week, when the adult arterial ramification is completed, are not clear, but longitudinal communicating anastomoses appear dorsally, laterally and ventrally between the dorsal intersegmental arteries during this period. Among the dorsal anastomoses, relative to $S u$ as the main axial artery, the residual postcostal anastomosis in the cephalic direction develops into $V e^{2,6,15)}$ and the posttransverse anastomosis becomes $C p .{ }^{15)} \mathrm{We}$ believe that among the precostal anastomoses, those in the caudal direction develop into $I s^{6,15)}$ and those in the cephalic direction develop, according to Hamilton, ${ }^{15)}$ into the thyrocervical trunk. However, in this period, it becomes $\mathrm{Ca}$ to supply the spinal cord, after which Tif, $S s$ and $T c$ extend from $C a$ to their target organs (each item was studied relative to each artery). Tin, which is a ventral anastomotic chain, ${ }^{2,15)}$ extended from the primary $S u$ in an inferior direction in the earliest stage at days 4.8 (stage 25) of the incubating egg of the domestic fowl. ${ }^{42)}$ In the pig, ${ }^{45)}$ it appears at roughly the same period $(12-14 \mathrm{~mm}$ stage) as other anastomoses, but in humans, the sites of origin of this artery and $\mathrm{Ca}$ are thought to be extremely close, suggesting that a common trunk is formed from both arteries in rare cases (Type II of Fig. 9). According to the report of Igarashi et al., ${ }^{20)}$ the lateral anastomotic chain develops into the lateral costal branch appearing with an incidence of $10-20 \%$.

The diverse formation of $S u$ branches appearing in adults derives from the residual state or nature of disappearance of the sites of origin of the abovedescribed arteries and their communicating anastomoses in the initial stage of development, and this formation varies between races, individuals as well as right and left sides.

In recent years, extensive use has been made in the fields of plastic and orthopedic surgery of a musculocutaneous flap consisting of the trapezius muscle supplied by the subclavian artery and the sternocleidomastoid muscle, and a compound flap formed by combining the above-mentioned structures with the bone to which they adhere. Demergasso ('79) ${ }^{14}$ ) reported grafting the upper and middle portions of the trapezius muscle in the restoration of large deficient portions of the oral cavity, oropharynx, hypopharynx and other structures. Our previous study ${ }^{43}$ ) reported that in all cases studied, the superficial branch of the transverse cervical artery $(R s)$ supplied almost all of this region, while only a small region (approximately $5 \%$ of the entire muscle) was supplied by the suprascapular artery (in approximately $70 \%$ of all cases studied) and the occipital artery (approximately $30 \%$ of all cases studied). Demergasso described the branch supplying the upper and middle portions as the superficial transverse cervical artery, but did not address the question of the origin of $R s$, which supplies these regions. Therefore, it should be remembered that, as shown in the above-described Fig. 6, Rs arises as a branch of $T t c$ from the 1st part of $S u$, in Type $c$ $(50-60 \%)$, which is common in whites and negroes, and in Type $a$, which is common in Asians (approximately $60 \%$ ), but Types $b$ and $d$, in which $R s$ arises from the 3 rd part, appeared with an incidence of $10 \%$ of our cases. In the reports of Siemssen ${ }^{40)}$. Ariyan $^{3)}$, and others, ${ }^{24,31)}$ who used the sterno- 
cleidomastoid muscle in the deficient parts of the floor of the mouth, tongue, oropharynx, and mandible, there are incorrect references to $\operatorname{Tin}^{40)}$ as the artery feeding the lower portion of this muscle, and that a branch from $S u$ does not supply the lower portion of this muscle. ${ }^{31)}$ However, Sato et al. $\left({ }^{\prime} 84\right)^{37)}$ reported that a branch $(C a, T c$ or $S s)$ from Ttc, supplied approximately $15 \%$ of entire region of this muscle in $75 \%$ of their subjects. On the basis of these considerations, we urge that due care be taken in the description of the ramification morphology at the site of $T t c$ origin.

\section{Acknowledgements}

The authors wish to thank the Misses Y. Emura and T. Kurihara of our Department of Anatomy for their helpful technical and secretarial assistance.

\section{References}

1) Adachi, B.: Das Arteriensystem der Japaner. I. 135-196, Kenkyusha, Tokyo, 1928.

2) Arey, L. B.: Developmental anatomy. 7th ed. 350-360, W B Saunders Co., Philadelphia and London, 1965.

3) Ariyan, S.: One-stage reconstruction for defects of the mouth using a sternomastoid myocutaneous flap. Plast. Reconstr. Surg., 63: 618-625, 1979.

4) Azuma, S.: On the arterial supply in the human deltoid muscle. J. Kyorin Med. Soc., 19: 15-34, 1988. (in Japanese)

5) Bardeen, C. R. and Lewis, W. H.: Development of the limbs, body-wall and back in man. Am. J. Anat., 1: 1-35, 1901.

6) Barry, A.: The aortic arch derivatives in the human adult. Anat. Rec., 111: 221-238, 1951.

7) Bean, R. B.: A composite study of the subclavian artery in man. Am. J. Anat., 4: 303-328, 1905.

8) Boyd, G. I.: Abnormality of subclavian artery associated with presence of the scalenus minimus. J. Anat., 68: 280-281, 1934.

9) Cavdar, S. and Arisan, E.: Variations in the extracranial origin of the human vertebral artery. Acta Anat., 135: 236-238, 1989.

10) Congdon, E. D.: Transformation of the aortic - arch system during the development of the human embryo. Contrib. Embryol., 68: 47-110, 1922.

11) Curnow, J.: Right subclavian artery passing in front of the scalenus anticus, Proc. Anat. Soc. of Great Britain and Ireland. J. Anat. Physiol., 22: V-Vi, 1888.

12) Daseler, E. H. and Anson, B. J.: Surgical anatomy of the subclavian artery and its branches. Surg. Gynecol. Obstet., 108: 149-174, 1959.

13) De Garis, C. F.: Patterns of branching of the subclavian artery in white and negro stocks. Am. J. Phys. Anthropol., 7: $95-107,1924$.

14) Demergasso, F. and Piazza, M. V.: Trapezius myocutaneous flap in reconstructive surgery for head and neck cancer: An original technique. Am. J. Surg., 138: 533-543, 1979.

15) Hamilton, W. J. and Mossman, H. W.: Human embryology. 4th cd. 268-272, Williams \& Wilkins Co., Baltimore, 1972.
16) Hepburn, D.: The lateral thoracic branch of the internal mammary artery. J. Anat. Physiol., 33: 259-260, 1899.

17) Huelke, D. F.: A study of the transverse cervical and dorsal scapular arteries. Anat. Rec., 132: 233-245, 1958.

18) Huelke, D. F.: The dorsal scapular artery - A proposed term for the artery to the rhomboid muscles. Anat. Rec., 142: $57-61,1962$.

19) Hunt, J. A.: Splitting of the scalenus anterior muscle by the roots of the brachial plexus and subclavian artery, as seen in the Bantu. Leech, 26: 23-25, 1956.

20) Igarashi, J., Kanbayashi, T., Yokoyama, T., Moriya, A., Takafuji, T. and Sato, Y.: Studies on the human R. costalis lateralis (Lateral mammary artery). J. Kyorin Med. Soc., (in Japanese)

21) Kagi, J.: Beitrag zur Topographie der Arteria transversa colli. Eine Untersuchung an 134 Halshalften. Anat. Anz., 107: $168-186,1959$.

22) Krechowiecki, A., Daniel, B. and Wiechowski, S.: Variation of the internal thoracic artery. Folia Morphol., 32: 173-184, 1973.

23) Lamberty, B. G. H.: The supra - clavicular axial patterned flap. Br. J. Plast. Surg., 32: 207-212, 1979.

24) Larson, D. L. and Goepfert, H.: Limitations of the sternocleidomastoid musculocutaneous flap in head and neck cancer reconstruction. Plast. Reconstr. Surg., 70: 328-332, 1982.

25) Lee, I. N.: Anomalous relationship of the inferior thyroid artery. Anat. Rec., 122: 499-506, 1955.

26) Lewis, W. H.: The development of the arm in man. Am. J. Anat., 1: 145-183, 1902.

27) Miyashita, K.: Artery of Chinese. Subclavian artery and its branches, 7. Manshu Igaku Zassi, 23: 879-896, 1935. (in Japanese)

28) Mori, Y.: Über die A. subclavia and ihre Aste bei japanischen Feten. Acta Anat. Nippon, 17: 229-255, 1941. (in Japanese)

29) Nizankowski, C., Noczynski, L. and Suder, E.: Variability of the origin of ramification of the subclavian artery in humans (studies on the Polish population). Folia Morphol., 41: 281-294, 1982.

30) Outi, H.: Studies on the transverse cervical and the suprascapular arteries. Acta Anat. Nippon, 38: I -2, 1963. (in Japanese)

31) Parkash, S., Ramakrishnam, K. and Ananthakrishnan, N.: Sternomastoid based island flap for lining after resection of oral carcinomas. Br. J. Plast. Surg., 33: 115-118, 1980.

32) Poker, N., Finby, N. and Steinberg, I.: The subclavian arteries: Roentgen study in health and disease. Am. J. Roentgenol., 80: 193-216, 1958.

33) Read, W. T. and Trotter, M.: The origins of transverse cervical and of transverse scapular arteries in American whites and negroes. Am. J. Phys. Anthropol., 28: 239-247, 1941.

34) Rohlich, K.: Über die Arteria transversa colli des Menschen. Anat. Anz., 79: 37-52, 1934.

35) Röhlich, K.: Über den Truncus thyreocervicalis des Menschen. Anat. Anz., 90: 129-148, 1940.

36) Saadeh, F. A.: The suprascapular artery: Case report of an unusual origin. Anat. Anz., 145: 83-86, 1979.

37) Sato, Y., Takeuchi, R., Kawashima, T., Takafuji, T. and Tozawa, T.: On the arterial supply in the human sternocleidomastoid muscle. J. Kyorin Med. Sco., 15: 39-55, 1984. (in Japanese)

38) Serizawa, M.: On the $m$. scalenus anterior in adult Japanese. J. Nippon Med. Sch., 35: 188-202, 1968

39) Shore, L. R.: An example of the muscle scalenus minimus. J. Anat., 60: 418-419, 1926. 
40) Siemssen, S. O., Kirkby, B. and O'Connor, T. P. F.: Immediate reconstruction of a resected segment of the lower jaw, using a compound flap of clavicle and sternomastoid muscle. Plast. Reconstr. Surg., 61: 724-735, 1978.

41) Stott, C. F.: A note on the scalenus minimus muscle. J. Anat., 62: 359-361, 1928.

42) Suzuki T.: Development of the subclavian artery in the chick, studied by injection method. Acta Anat. Nippon, 62: 556-580, 1987. (in Japanese)

43) Takafuji, T.: On the arterial supply in the human trapezius muscle. Acta Anat. Nippon, 59: 110-121, 1984. (in Japanese)
44) Thomson, A.: Second annual report of the committee of collective investigation of the anatomical society of Great Britain and Ireland for the year 1890-91. J. Anat. Physiol.. 26: 76-93, 1891.

45) Woollard, H. H.: The development of the principal arterial stems in the forelimb of the pig. Contrib. Embryol., 70: 139-154, 1922.

46) Yamada, M. and Mannen, H.: Anatomy for dissections. 25-28, Nankodo, Tokyo, 1985. (in Japanese)

47) Yamada, S.: Statistische Beobachtung uber die Arteria mammaria (interna) lateralis. Acta Anat. Nippon, 8: $658-672,1935$. (in Japanese) 